\title{
El talento humano, una perspectiva desde la gerencia en las mipymes ${ }^{1}$
}

Juan Sebastián Dugarte-Mendoza ${ }^{3}$

Corporación Universitaria Minuto de Dios - Uniminuto

Vicerrectoría Santanderes, Centro Regional Bucaramanga mdugarte@uniminuto.edu.co

Yohanna Milena Rueda-Mahecha ${ }^{4}$ Corporación Universitaria Minuto de Dios - Uniminuto Vicerrectoría Santanderes, Centro Regional Bucaramanga yruedamahec@uniminuto.edu.co

Fecha de recepción: 07 de junio de 2019

Fecha de aprobación: 17 de noviembre de 2020

Fecha de publicación: 17 de marzo de 2021

Cómo citar este artículo / To reference this article / Comment citer cet article / Para citar este artigo:

Silva-Giraldo, C. A.; Dugarte-Mendoza, J. S.; Rueda-Mahecha, Y. M. (2020). El talento humano, una perspectiva desde la gerencia en las mipymes. Revista Escuela de Administración de Negocios, (89), 13-32.

DOI: https://doi.org/10.21158/01208160.n89.2020.2814

\section{Resumen}

El estudio que da origen a este artículo se realizó con el fin de analizar la percepción de la gerencia con relación a la gestión del talento humano en las mipymes. Para el análisis se utilizó una investigación de tipo descriptiva con un enfoque mixto y un diseño transversal no experimental; y se estableció como población objeto de estudios a los propietarios, gerentes y directivos de las mipymes ubicadas en los municipios de Bucaramanga, Floridablanca, Girón y Piedecuesta del departamento de Santander, Colombia. Finalmente se hizo una selección de muestra poblacional de mil veinticinco individuos que ocupan los cargos establecidos

1 Derivado del proyecto de investigación «Estudio sobre la gestión del talento humano en las Mipymes de Santander, Colombia». Corporación Universitaria Minuto de Dios- Uniminuto Centro Regional Bucaramanga.

2 Administrador de Empresas, Universidad Nacional Abierta y a Distancia UNAD. Especialista en Gestión de Proyectos, Universidad Nacional Abierta y a Distancia UNAD. MBA - Master especializado en Comercio Internacional, Cerem Business School. Magíster en Paz, Desarrollo y Ciudadanía, Uniminuto. Doctorando en Ciencias Económicas y Administrativas, UCIMEXICO. Integrante del Grupo de Investigación en Desarrollo Humano, Tejido Social e Innovaciones Tecnológicas (GIDTI) y QUANTUM. ORCID: https://orcid.org/0000-0002-8023-8531

3 Ingeniero Mecánico, Universidad Pontificia Bolivariana. MBA - Magister en Administración de Empresas con Especialidad en Dirección de Proyectos, Universidad de Viña del Mar - Chile. Doctorando en Dirección de Proyectos, Universidad Benito Juárez, México. Integrante del grupo QUANTUM. ORCID: https://orcid.org/0000-0003-1149-0503

4 Fonoaudióloga, Corporación Universitaria Iberoamericana. Especialista en Administración en Salud Ocupacional, Universidad Jorge Tadeo Lozano, Magíster en Educación con énfasis en Lectura, Escritura y Matemáticas, Universidad Jorge Tadeo Lozano. Investigador del grupo de Investigación en Desarrollo Humano, Tejido Social e Innovaciones Tecnológicas (GIDTI) y QUANTUM. ORCID: https://orcid.org/0000-0002-0722-6593 
dentro de la población objeto. Destacando factores positivos y negativos que se dan en la gestión del personal, e impactan la mejora de la relación entre las directivas y colaboradores, se pudo establecer una serie de postulados y supuestos de acuerdo a las variables analizadas. Además, teniendo en cuenta los estudios consultados y los resultados, se resalta que gran parte de la relación entre las partes se define a partir de la visión de responsabilidad, lealtad, capacidad, clima laboral, condición salarial y participación de los colaboradores en las decisiones de la organización, lo que en su conjunto permite mejorar las condiciones de efectividad y eficiencia organizacional y por tanto, su competitividad.

Palabras clave: talento humano; gestión de talento humano; gestión de personal; gerencia de talento humano; gerencia de mipymes; eficiencia organizacional.

\title{
Human talent, a perspective from management in SMEs
}

\begin{abstract}
The study that gives rise to this article was conducted with the purpose of analyzing the perception of managers in relation to the management of human talent in SMEs. For the analysis, we used a descriptive research with a mixed approach and a non-experimental cross-sectional design; with a target population composed of owners, managers, and directors of SMEs in the municipalities of Bucaramanga, Floridablanca, Girón and Piedecuesta in the department of Santander, Colombia. Finally, the selected population was a sample of 1,025 individuals occupying the positions established within the target population. Highlighting the positive and negative factors that take place in personnel management and that impact the improvement of the relationship between managers and collaborators, and according to the variables that were analyzed, it was possible to establish a series of postulates and assumptions. In addition, taking into account the studies consulted and their results, it is feasible to highlight that a large part of the relationship between the parties is based on the vision of responsibility, loyalty, capacity, work environment, salary conditions, and participation of the collaborators in the decisions that are made within the organization, all this allows the improvement of the conditions of organizational effectiveness and efficiency and, therefore, competitiveness.
\end{abstract}

Keywords: human talent; human talent management; personnel management; human talent administration; SME management; organizational efficiency.

\section{Talento humano, uma perspectiva a partir da gestão nas MPME}

\section{Resumo}

O estudo que dá origem a este artigo foi realizado com o objetivo de analisar a percepção da gerência em relação à gestão do talento humano nas MPME. Para a análise, foi utilizada uma pesquisa descritiva com abordagem mista e desenho transversal não experimental e foram estabelecidos como população-alvo dos estudos os proprietários, gerentes e diretores das MPME localizadas nos municípios de Bucaramanga, Floridablanca, Girón e Piedecuesta no departamento de Santander, Colômbia. Finalmente, foi feita a seleção de uma amostra populacional de mil e vinte e cinco indivíduos que ocupam os cargos estabelecidos na população-alvo. Destacando fatores positivos e negativos que ocorrem na gestão de pessoas e impactam 
na melhoria do relacionamento entre diretores e colaboradores, foi possível estabelecer uma série de postulados e hipóteses de um acordo com as variáveis analisadas. Além disso, tendo em conta os estudos consultados e os resultados, destaca-se que grande parte da relação entre as partes se define a partir da visão de responsabilidade, lealdade, competência, ambiente de trabalho, posição salarial e participação dos colaboradores nas decisões da organização, o que, no seu conjunto, permite melhorar as condições de eficácia e eficiência organizacional e, consequentemente, a sua competitividade.

Palavras-chave: talento humano, gestão de talentos humanos; gestão de pessoas; gerência de talentos humanos; gestão de MPME; eficiência organizacional.

\section{Ressources humaines : perspective managériale dans les micro-PME colombiennes}

\section{Résumé}

Cette étude tentera d'analyser la perception de la gestion des ressources humaines dans les micro-PME. Il s'agit d'une recherche de type descriptif, d'approche mixte, transversal et non expérimental. Des propriétaires, gérants ou directeurs de micro-PME situées dans les villes de Bucaramanga, Floridablanca, Girón et Piedecuesta du département de Santander ont été pris comme population cible de l'étude. Cette sélection a été réalisée sur la base d'un échantillon de mille vingt-cinq personnes occupant les fonctions prédéfinies au sein de cette population cible. A partir d'une mise en évidence de facteurs positifs et négatifs intervenant dans la gestion du personnel et ayant un impact sur l'amélioration de la relation entre managers et collaborateurs, cet article établit une série de postulats et d'hypothèses sujets aux variables d'analyses. D’autre part, et compte tenu des résultats d'études consultées, il est possible de mettre en évidence qu'une grande partie de la relation entre les acteurs se définit au travers de la responsabilité, de la loyauté, de l'environnement de travail, du statut salarial ou de la participation des salariés aux décisions de l'entreprise et doit permettre d'améliorer les conditions d'efficacité et d'efficience organisationnelle et donc, la compétitivité des entreprises.

Mots-clés: ressources humaines; gestion des ressources humaines; gestion du personnel; gestion des microPME; efficacité organisationnelle. 


\section{Introducción}

$\mathrm{E}$ n las empresas colombianas se viven grandes cambios de forma acelerada, en particular, la competencia y el aumento de la demanda especializada, llevan a la generación de cambios significativos en la forma como se gestiona el talento humano como parte fundamental en los procesos y el crecimiento organizacional, generando la reformulación de los objetivos, metas y estrategias; y finalmente a la toma de decisiones.

De acuerdo con Rangel-Pico, Silva-Giraldo, Nino-Liévano y Pérez-Olmos (2017), en Colombia según la Ley 905 de 2004, que modificó la Ley 590 de 2000, se entiende por mipymes a las micro, incluidas las famiempresas, pequeña y mediana empresa, como toda unidad de explotación económica, realizada por persona natural o jurídica, en actividades empresariales, agropecuarias, industriales, comerciales o de servicio que se encuentra en el área rural o urbana. Hoy en día, un porcentaje del sector productivo en Colombia está conformado por mipymes representando el $96 \%$ y aportando el $40 \%$ al PIB, siendo parte activa del desarrollo y el crecimiento del país.

En este orden de ideas, las micro, pequeñas y medianas empresas se han venido convirtiendo en las principales protagonistas del desarrollo en las diversas regiones en Colombia, teniendo un impacto en los diversos indicadores macroeconómicos. Como lo exponen Celis-Salazar y Silva-Giraldo (2018) de acuerdo a cifras aportadas por el DANE a inicio del 2018 , las mipymes generan alrededor de $67 \% \mathrm{del} \mathrm{em-}$ pleo, de igual manera el registro único empresarial y social (RUES), establece que en el país el 94,7 \% de las empresas registradas son microempresas y $4,9 \%$ pequeñas y medianas.
Para el caso de la investigación, en el área metropolitana de Bucaramanga, se establece, de acuerdo con el observatorio de Competitividad, que el 99,7 \% de empresas creadas de acuerdo al tamaño son micro, el $0,03 \%$ medianas y el $0,01 \%$ pequeñas, mostrado el grado de importancia en el desarrollo en la región (Celis-Salazar y Silva-Giraldo, 2018).

Por otra parte, investigaciones destacan que muchos de los cambios que se viven en las mipymes, se generan por sus directivas, apoyadas por los colaboradores, siendo estos últimos el recurso más importante de dichas organizaciones (Enciso y Porras-Jiménez, 2011).

Teniendo en cuenta lo anterior, se puede establecer que la competitividad de las mipymes se debe en un alto porcentaje a la calidad del talento humano, de tal forma que son las competencias de los colaboradores y su desarrollo las que llegan a convertirse en una ventaja competitiva, constituyéndose en un aspecto fundamental para las organizaciones que lleva a designar recursos y a desarrollar las habilidades y destrezas de los trabajadores para la consecución de los objetivos estratégicos de la empresa (Mejía-Giraldo, Bravo-Castillo y Montoya-Serrano, 2013).

En este orden de ideas, la gestión del talento humano ha venido cambiando a través del tiempo, pasando de un departamento de recursos humanos, cuyo enfoque era el de funciones de contratación, nómina y beneficios, a lo que hoy en día es, un área que se encarga de comprender el impacto positivo de los colaboradores en el crecimiento empresarial; es por ello, que su preocupación se centra en la generación de equipos de empleados calificados, que se comprometen e involucran con las metas de la organización (Enciso y Porras-Jiménez, 2011). 
Continuando, el sistema de gestión del talento humano en las mipymes busca medir y administrar el desempeño de los colaboradores, por medio de procesos de capacitación, retroalimentación y apoyo, permitiendo generar una visión más clara de las competencias que se necesitan para alcanzar el éxito.

Adicionalmente, busca generar un ambiente laboral apropiado, que fomenta la productividad por medio del compromiso y la motivación, identificando así las necesidades de las personas para encaminar las metas y los objetivos individuales como colectivos entre colaboradores y organización.

Como lo establecen Silva-Giraldo, Dugarte-Mendoza y Rueda-Mahecha (2020), tener a los mejores colaboradores significa obtener los mejores rendimientos, convirtiéndose en una ventaja competitiva con relevancia e importancia. El mantener a través del tiempo a los colaboradores se puede percibir en los costos organizacionales en el largo plazo; esto se soporta en que la realización de nuevas contrataciones repercute en cifras en casi tres veces mayores en el costo de retener a un buen colaborador que se convierte en un activo valioso.

Adicionalmente, la adaptación de un nuevo colaborador en una organización puede llegar a tardar hasta seis meses, desde el momento de la incorporación hasta que alcance el nivel de productividad que se necesita para demostrar unas habilidades y destrezas esperadas de acuerdo al puesto de trabajo (Rangel-Pico, et al., 2017).

De igual manera, integrar a una persona en la cultura organizacional de la empresa puede llegar a tardar hasta dieciocho meses. Todo este proceso se hace aún más largo según la estrategia y la actividad de la empresa, pudiendo necesitarse hasta veinticuatro meses para que el nuevo colaborador que ingresa a la organización se integre de forma adecuada (Rangel-Pico, et al., 2017).

Todo lo anterior, no se podría llegar a dar si no se logra generar un espacio colectivo y de trabajo colaborativo que lleve a lo que conocemos como clima laboral, el cual influye directamente con la satisfacción de los colaboradores, ayudando a mejorar la productividad.
Por lo tanto, si se presenta un buen ambiente laboral se va a presentar una orientación hacia los objetivos laborales, un mal clima en la organización puede llegar a destruir el trabajo, esto ocasionado por situaciones de conflicto o malestar de los empleados que pueden afectar el rendimiento (Jaramillo-Naranjo, 2005).

En este orden de ideas, la calidad del clima laboral se encuentra muy relacionada con el manejo del objeto social de los directivos, estableciendo el comportamiento que se da hacia los trabajadores y la manera en la que se relacionan, las interacciones que tienen con la empresa, así como las herramientas que se utilizan y las características propias de los empleados.

En consecuencia, se infiere que será un rol de la gerencia, el lograr una interacción entre empleados y las condiciones que exige el mercado y la competitividad organizacional, sin perder el desarrollo integral del equipo de trabajo, el cual apoya en los procesos de toma de decisiones, gracias al conocimiento en áreas específicas.

Según Chiavenato (2007), se debe partir del análisis de las relaciones entre individuos y organizaciones para propiciar la interacción de la organización y los grupos de personas que hacen parte de la misma y la formación de sistemas abiertos, siendo estos los que describen las acciones e interacciones que se dan en los organismos que se rodean en el contexto de la organización y lo que se conoce como cultura organizacional.

Como apoyo a lo anterior, Alles (2005), plantea que el elemento de mayor relevancia en la formación de equipos de trabajo es la participación en los procesos de toma de decisiones, permitiendo aprovechar el conocimiento y experticia de los colaboradores, generando procesos basados en la razón y con un horizonte global. 
Adicionalmente, de acuerdo con Genesi y Suarez (2010), la gestión del talento permite generar un ambiente para la interacción entre empleados y decisiones, además, cuando la gente participa en la toma de la decisión, se siente más comprometida a lograr los resultados deseados, llegando a convertirse en un motivador relevante en pro de los trabajadores.

Por otra parte, Baguer (2009) resalta que los cambios que se han presentado en los departamentos de talento humano han permitido desarrollar un bienestar laboral, aumentar los nuevos talentos y motivar a los colaboradores. Si la empresa tiene un programa de bienestar laboral efectivo, podrá hacer una relación de la eficiencia y productividad.

Lo anterior, abre paso a Ramírez-Lira y Dávila-Ibarra (2018), quienes plantean que cuando se involucra la socialización y el compañerismo desde lo que el líder enseña, transmite y establece como ciertas normas y comportamientos que responderán a las demandas y los conflictos de la empresa, se determina la productividad, la competitividad y la calidad de vida de la organización y de los sujetos que la conforman.
De otra parte, Aranda-Leyton (2016) hace énfasis en que en la empresa moderna se trabaja en comunidad, es decir, se comunica todo el colectivo, se crean lazos de amistad y compañerismo, llegando a aprovechar las habilidades y destrezas de cada uno de los miembros de la organización, creando una cultura donde todos comparten los objetivos y metas.

Finalmente, Angulo (2006) afirma que invertir en las personas puede generar grandes beneficios, siendo el recurso humano el que es capaz de potenciar el trabajo en equipo y transformar la organización radicalmente, permitiendo de manera individual y grupal, y por medio de una capacitación direccionada en las necesidades reales y en competencias, el crecimiento de la organización.

Ahora bien, el desarrollo de la presente investigación pretende abordar los elementos expuestos en el marco de las mipymes ubicadas en Bucaramanga y su área metropolitana, todo ello visto desde la perspectiva de la gerencia, lo que permitirá corroborar la relación entre los conceptos abordados, y la situación que se vive en la práctica del hacer empresa en Colombia.

\section{Metodología}

$\mathrm{E}^{\mathrm{l}}$ estudio se desarrolló por medio de una nvestigación de tipo descriptivo con un enfoque mixto y un diseño transversal no experimental, buscando analizar con ello la percepción y aplicación de prácticas en torno a la gestión del talento humano en las mipymes.

Para ello se estableció como población objeto de estudio a los propietarios, gerentes y directivos de las mipymes ubicadas en los municipios de Bucaramanga, Floridablanca, Girón y Piedecuesta del departamento de Santander, Colombia. Haciendo una selección de muestra poblacional de mil veinticinco individuos que ocupan los cargos establecidos dentro de la población objeto.
La recolección de datos se hizo por medio de la técnica de la encuesta, recabando información con relación a los datos de las empresas y los gerentes o directivos, teniendo presente para ello la ubicación, actividad económica, año de creación, existencia de reglamento de trabajo y número de empleados.

De igual manera, se establecieron las variables de análisis de la investigación de acuerdo a los factores de medición que permiten evaluar el desempeño laboral desde un punto de vista cualitativo y cuantitativo, relacionando entre ellas la percepción de la honestidad, la responsabilidad, la lealtad, la perspectiva de capacidad, el clima laboral, condiciones salariales, mejora de productividad, evaluación de satisfacción laboral, implementación de programas 
que buscan la mejora del bienestar, condiciones de evaluación de desempeño de empleados, e interacción empleados con los procesos de toma de decisiones.

Las etapas de la investigación, presentan como primer paso, la identificación de las variables según el instrumento seleccionado; acto seguido se procede a aplicar elementos de estadística descriptiva, con el fin de reconocer el entorno y las prácticas gerenciales. Finalmente se establecen una serie supuestos e hipótesis con el fin de observar la asociación de variables y validar la aplicación de teorías entorno a la gestión del talento humano.

El instrumento elaborado presenta la siguiente estructura; la cual fue diseñada con el objetivo de reconocer tres áreas principales: a) Características de la organización, b) Percepciones de la gerencia y c) Acciones de la gerencia. Cada una de ellas presenta las siguientes preguntas:

Tabla 1. Presentación del instrumento

\begin{tabular}{|c|c|}
\hline \multicolumn{2}{|r|}{ Presentación y análisis del instrumento } \\
\hline Área de estudio & Pregunta asociada \\
\hline \multirow{4}{*}{ Características de la organización } & Ubicación de la empresa \\
\hline & ¿Cuál es la actividad principal de la empresa? \\
\hline & ¿Cuál es el año de fundación de la empresa? \\
\hline & ¿Existe un reglamento de trabajo y el mismo es de conocimiento de los empleados? \\
\hline \multirow{7}{*}{$\begin{array}{l}\text { Percepciones del talento humano, desde } \\
\text { la gerencia }\end{array}$} & ¿Cuántos empleados tienen la empresa? \\
\hline & ¿Considera que los empleados de la empresa son honestos? \\
\hline & ¿Considera que los empleados de la empresa son responsables? \\
\hline & ¿Considera que los empleados de la empresa son leales? \\
\hline & ¿Considera que la empresa tiene empleados capaces? \\
\hline & ¿Considera que en la empresa se maneja un buen clima laboral y todos tienen buen trato? \\
\hline & $\begin{array}{l}\text { ¿La empresa paga sueldos muy superiores a los de otras empresas que desarrollan el } \\
\text { mismo objeto social? }\end{array}$ \\
\hline \multirow{5}{*}{ Acciones ejecutadas por la gerencia } & $\begin{array}{l}\text { ¿Como gerente de la organización me enfoco en mejorar la productividad de los } \\
\text { empleados? }\end{array}$ \\
\hline & ¿En la empresa se evalúa la satisfacción laboral de los empleados? \\
\hline & ¿En la empresa se implementan programas para mejorar el bienestar de los empleados? \\
\hline & ¿La empresa evalúa el desempeño de los empleados? \\
\hline & $\begin{array}{l}\text { ¿Los empleados son tenidos en cuenta para la toma de decisiones en los diferentes niveles } \\
\text { y cargos? }\end{array}$ \\
\hline
\end{tabular}

Fuente. Elaboración propia. 
Una vez, identificadas las principales categorías, se procede a determinar los factores asociados a cada una de las preguntas, y se codifican, esto con la finalidad de facilitar los procesos de análisis.

Tabla 2. Reconocimiento y clasificación de variables

\begin{tabular}{|c|c|c|c|}
\hline \multicolumn{4}{|c|}{ Cuadro de operacionalidad de variables } \\
\hline Código de variable & Pregunta asociada & Nombre de la variable & $\begin{array}{c}\text { Tipo de variable } \\
\text { (Cualitativa / Cuantitativa) }\end{array}$ \\
\hline V00 & Ubicación de la empresa & Ubicación & Cualitativa \\
\hline V01 & ¿Cuál es la actividad principal de la empresa? & Actividad económica & Cualitativa \\
\hline V02 & ¿Cuál es el año de fundación de la empresa? & Años & Cuantitativa \\
\hline V03 & $\begin{array}{l}\text { ¿Existe un reglamento de trabajo y el mismo es } \\
\text { de conocimiento de los empleados? }\end{array}$ & $\begin{array}{l}\text { Existencia de reglamento } \\
\text { de trabajo }\end{array}$ & Cualitativa \\
\hline V04 & ¿Cuántos empleados tiene la empresa? & Número de empleados & Cuantitativa \\
\hline V05 & $\begin{array}{l}\text { ¿Considera que los empleados de la empresa son } \\
\text { honestos? }\end{array}$ & Honestidad & Cualitativa \\
\hline V06 & $\begin{array}{l}\text { ¿Considera que los empleados de la empresa son } \\
\text { responsables? }\end{array}$ & Responsabilidad & Cualitativa \\
\hline V07 & $\begin{array}{l}\text { ¿Considera que los empleados de la empresa } \\
\text { son leales? }\end{array}$ & Lealtad & Cualitativa \\
\hline V08 & $\begin{array}{l}\text { ¿Considera que la empresa tiene empleados } \\
\text { capaces? }\end{array}$ & Capacidad & Cualitativa \\
\hline V09 & $\begin{array}{l}\text { ¿Considera que en la empresa se maneja un } \\
\text { buen clima laboral y todos tienen buen trato? }\end{array}$ & Clima laboral & Cualitativa \\
\hline V10 & $\begin{array}{l}\text { ¿La empresa paga sueldos muy superiores a los } \\
\text { de otras empresas que desarrollan el mismo } \\
\text { objeto social? }\end{array}$ & Salarios & Cuantitativa \\
\hline V11 & $\begin{array}{l}\text { ¿Como gerente de la organización me enfoco en } \\
\text { mejorar la productividad de los empleados? }\end{array}$ & Mejora de productividad & Cualitativa \\
\hline V12 & $\begin{array}{l}\text { ¿En la empresa se evalúa la satisfacción laboral } \\
\text { de los empleados? }\end{array}$ & $\begin{array}{l}\text { Evaluación de } \\
\text { satisfacción laboral }\end{array}$ & Cualitativa \\
\hline V13 & $\begin{array}{l}\text { ¿En la empresa se implementan programas para } \\
\text { mejorar el bienestar de los empleados? }\end{array}$ & $\begin{array}{l}\text { Programas para mejorar } \\
\text { el bienestar }\end{array}$ & Cualitativa \\
\hline V14 & $\begin{array}{l}\text { ¿La empresa evalúa el desempeño de los } \\
\text { empleados? }\end{array}$ & $\begin{array}{l}\text { Evaluación de } \\
\text { desempeño de } \\
\text { empleados }\end{array}$ & Cualitativa \\
\hline V15 & $\begin{array}{l}\text { ¿Los empleados son tenidos en cuenta para la } \\
\text { toma de decisiones en los diferentes niveles y } \\
\text { cargos? }\end{array}$ & $\begin{array}{l}\text { Interacción empleados y } \\
\text { decisiones }\end{array}$ & Cualitativa \\
\hline
\end{tabular}

Fuente. Elaboración propia.

\section{Desarrollo del contenido}

A continuación, se realiza una presentación del análisis realizado sobre cada una de las variables, partiendo de la aplicación de estadística descriptiva; los resultados se presentarán según el orden mencionado en la metodología. 
Figura 1. V00. Ubicación de las mipymes en el área metropolitana de Bucaramanga

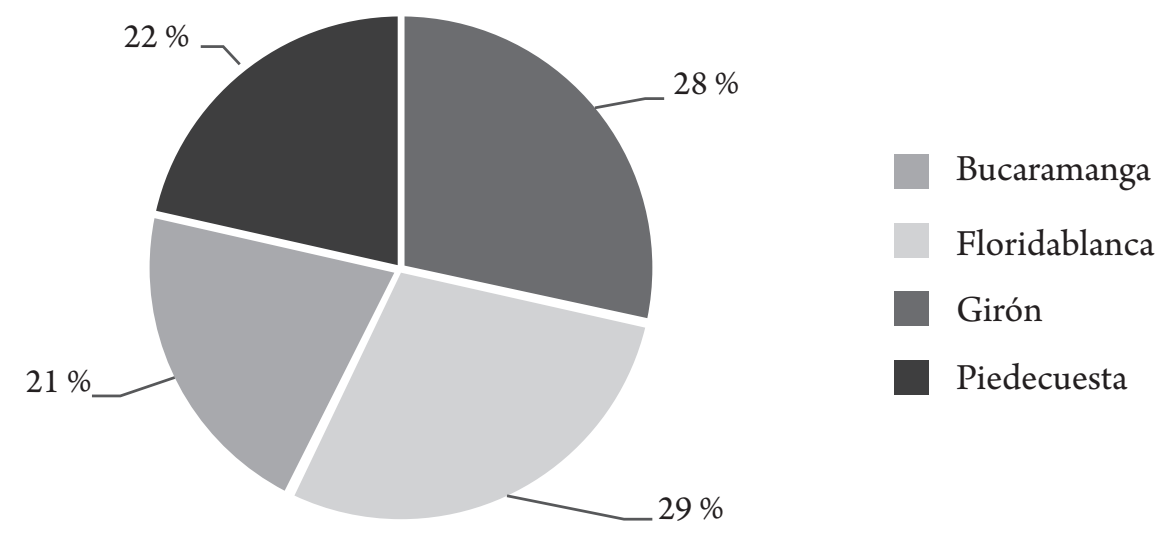

Fuente. Elaboración propia.

La presentación de la ubicación de las mipymes en Santander, permite identificar una adecuada distribución de los datos trabajados; es conveniente destacar que los municipios mencionados hacen parte de la denominada área metropolitana de Bucaramanga. Por tanto, se infiere una adecuada organización y homogenización de los datos.

Tabla 3. V01. Número de empresas por actividad principal

\begin{tabular}{|c|c|c|c|c|}
\hline \multicolumn{5}{|c|}{ V01 - Conteo, Número de empresas por actividad principal } \\
\hline Actividad económica & Número de empresas & Porcentaje & $\begin{array}{c}\text { Conteo acumulado } \\
\text { de empresas }\end{array}$ & $\begin{array}{c}\text { Porcentaje acumulado } \\
\text { de empresas }\end{array}$ \\
\hline 1 & 4 & 0,39 & 4 & 0,39 \\
\hline 9 & 18 & 1,76 & 22 & 2,15 \\
\hline 10 & 1 & 0,1 & 23 & 2,24 \\
\hline 12 & 2 & 0,2 & 25 & 2,44 \\
\hline 13 & 11 & 1,07 & 36 & 3,51 \\
\hline 14 & 4 & 0,39 & 40 & 3,9 \\
\hline 15 & 4 & 0,39 & 44 & 4,29 \\
\hline 19 & 8 & 0,78 & 52 & 5,07 \\
\hline 20 & 2 & 0,2 & 54 & 5,27 \\
\hline 25 & 1 & 0,1 & 55 & 5,37 \\
\hline 30 & 2 & 0,2 & 57 & 5,56 \\
\hline 31 & 5 & 0,49 & 62 & 6,05 \\
\hline 32 & 1 & 0,1 & 63 & 6,15 \\
\hline 34 & 1 & 0,1 & 64 & 6,24 \\
\hline 36 & 1 & 0,1 & 65 & 6,34 \\
\hline 37 & 1 & 0,1 & 66 & 6,44 \\
\hline 38 & 2 & 0,2 & 68 & 6,63 \\
\hline 39 & 2 & 0,2 & 70 & 6,83 \\
\hline 40 & 3 & 0,29 & 73 & 7,12 \\
\hline 41 & 5 & 0,49 & 78 & 7,61 \\
\hline
\end{tabular}




\begin{tabular}{|c|c|c|c|c|}
\hline 42 & 17 & 1,66 & 95 & 9,27 \\
\hline 43 & 6 & 0,59 & 101 & 9,85 \\
\hline 44 & 22 & 2,15 & 123 & 12 \\
\hline 46 & 8 & 0,78 & 131 & 12,78 \\
\hline 47 & 72 & 7,02 & 203 & 19,8 \\
\hline 48 & 60 & 5,85 & 263 & 25,66 \\
\hline 49 & 1 & 0,1 & 264 & 25,76 \\
\hline 51 & 10 & 0,98 & 274 & 26,73 \\
\hline 55 & 1 & 0,1 & 275 & 26,83 \\
\hline 56 & 9 & 0,88 & 284 & 27,71 \\
\hline 57 & 1 & 0,1 & 285 & 27,8 \\
\hline 58 & 9 & 0,88 & 294 & 28,68 \\
\hline 59 & 1 & 0,1 & 295 & 28,78 \\
\hline 60 & 90 & 8,78 & 385 & 37,56 \\
\hline 61 & 203 & 19,8 & 588 & 57,37 \\
\hline 62 & 7 & 0,68 & 595 & 58,05 \\
\hline 63 & 8 & 0,78 & 603 & 58,83 \\
\hline 64 & 20 & 1,95 & 623 & 60,78 \\
\hline 65 & 28 & 2,73 & 651 & 63,51 \\
\hline 66 & 1 & 0,1 & 652 & 63,61 \\
\hline 67 & 5 & 0,49 & 657 & 64,1 \\
\hline 68 & 3 & 0,29 & 660 & 64,39 \\
\hline 69 & 75 & 7,32 & 735 & 71,71 \\
\hline 70 & 96 & 9,37 & 831 & 81,07 \\
\hline 71 & 1 & 0,1 & 832 & 81,17 \\
\hline 72 & 12 & 1,17 & 844 & 82,34 \\
\hline 73 & 1 & 0,1 & 845 & 82,44 \\
\hline 76 & 2 & 0,2 & 847 & 82,63 \\
\hline 77 & 4 & 0,39 & 851 & 83,02 \\
\hline 79 & 54 & 5,27 & 905 & 88,29 \\
\hline 81 & 1 & 0,1 & 906 & 88,39 \\
\hline 82 & 1 & 0,1 & 907 & 88,49 \\
\hline 84 & 3 & 0,29 & 910 & 88,78 \\
\hline 85 & 3 & 0,29 & 913 & 89,07 \\
\hline 89 & 1 & 0,1 & 914 & 89,17 \\
\hline 91 & 2 & 0,2 & 916 & 89,37 \\
\hline 93 & 1 & 0,1 & 917 & 89,46 \\
\hline 96 & 10 & 0,98 & 927 & 90,44 \\
\hline 97 & 11 & 1,07 & 938 & 91,51 \\
\hline 99 & 3 & 0,29 & 941 & 91,8 \\
\hline 100 & 1 & 0,1 & 942 & 91,9 \\
\hline 103 & 1 & 0,1 & 943 & 92 \\
\hline 105 & 2 & 0,2 & 945 & 92,2 \\
\hline 106 & 19 & 1,85 & 964 & 94,05 \\
\hline
\end{tabular}




\begin{tabular}{|c|c|c|c|c|}
\hline 107 & 4 & 0,39 & 968 & 94,44 \\
\hline 109 & 4 & 0,39 & 972 & 94,83 \\
\hline 111 & 3 & 0,29 & 975 & 95,12 \\
\hline 112 & 2 & 0,2 & 977 & 95,32 \\
\hline 114 & 1 & 0,1 & 978 & 95,41 \\
\hline 115 & 42 & 4,1 & 1020 & 99,51 \\
\hline 117 & 2 & 0,2 & 1022 & 99,71 \\
\hline 141 & 1 & 0,1 & 1023 & 99,8 \\
\hline 239 & 1 & 0,1 & 1024 & 99,9 \\
\hline 755 & 1 & 0,1 & 1025 & 100 \\
\hline $\mathrm{N}=$ & 1025 & & & \\
\hline
\end{tabular}

Fuente. Elaboración propia.

En términos del número de empresas por actividad principal, las de mayor relevancia para la investigación son las que se encuentran dentro de lo siguientes códigos y actividades:

- Código 44: Venta, mantenimiento y reparación de motocicletas y sus partes, piezas y accesorios.

- Código 47: Venta al mayoreo de alimentos, bebidas y tabaco.

- Código 48: Venta al mayoreo de ropa, calzado y textiles.

- Código 60: Venta al menudeo en comercios no especializados.

- Código 61: Venta al menudeo de alimentos, bebidas y tabaco en comercios especializados-no restaurantes-.
- Código 65: Venta al menudeo de ferretería, pinturas y productos de vidrio en comercios especializados.

- Código 69: Venta al por menor de otros productos en comercios especializados.

- Código 70: Venta al por menor en puestos de venta y mercados.

- Código 79: Actividades de servicio de comidas y bebidas.

- Código 115: Otras actividades de servicios personales.

Figura 2. V02. Año de fundación de la empresa

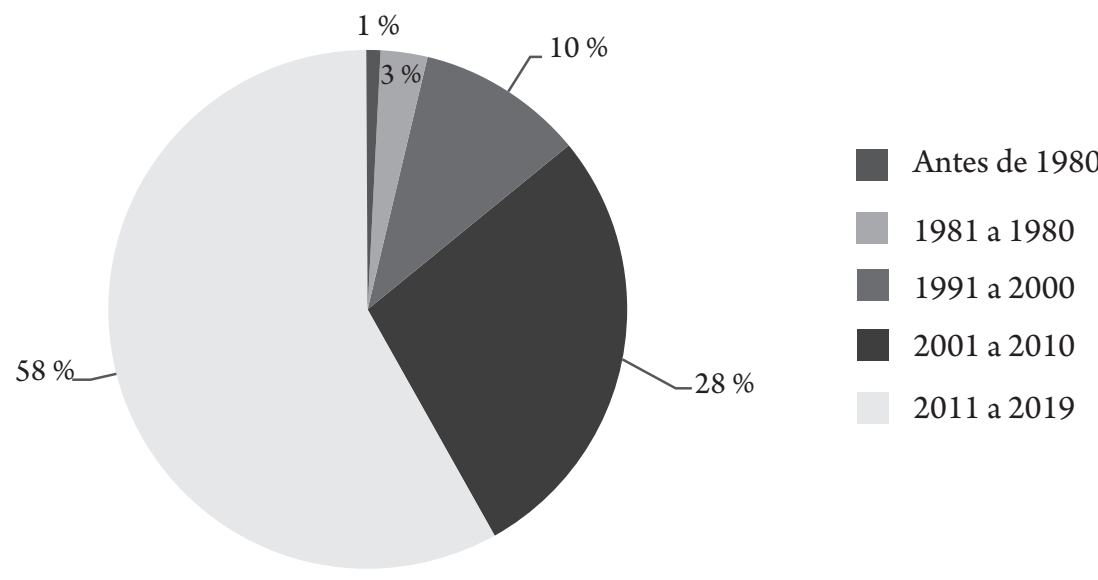

Fuente. Elaboración propia. 
Por otra parte, el año de fundación de la empresa, permite observar la distribución según el año de fundación de las empresas; se destaca que el $58 \%$ corresponden a mipymes creadas en el periodo 2011 a 2019; seguido de un $28 \%$ de aquellas creadas entre 2001 y 2010.

Figura 3. V03. Existencia de reglamento de trabajo y reconocimiento por parte de los empleados

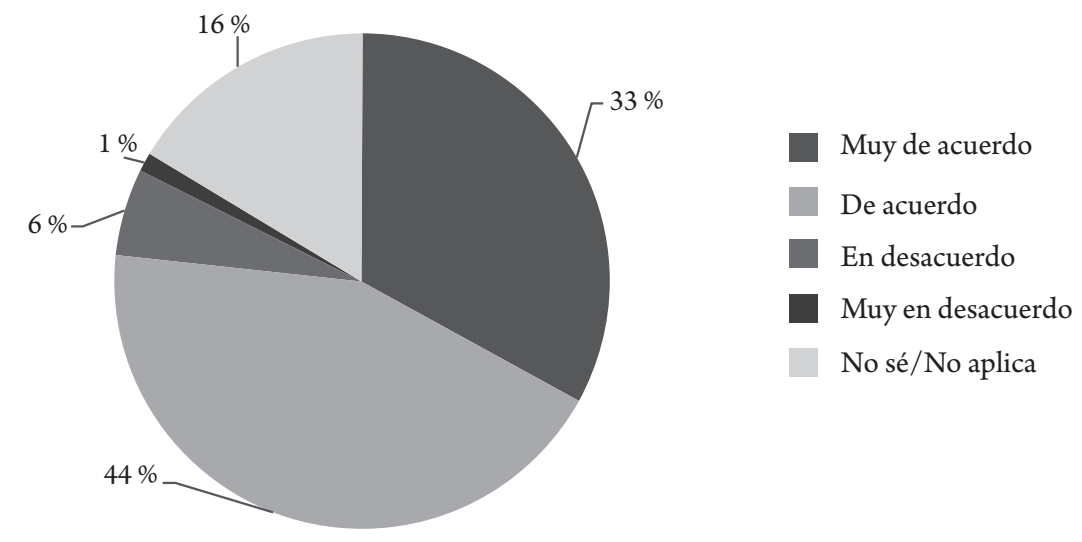

Fuente. Elaboración propia.

Respecto a V03 Existencia de reglamento de trabajo y conocimiento por parte de los empleados se evidencia que en el $84 \%$ de las empresas existe un reglamento de trabajo, y sobre dicho porcentaje el $77 \%$ de los gerentes reconocen que existe un adecuado reconocimiento del mismo por parte de los colaboradores.

Tabla 4. V04. Número de empleados por empresa
Es posible apreciar el número de empleados por empresa, de ello se destaca que el 95,8 \% de los encuestados pueden ser clasificados como microempresas ya que presentan un número inferior a 10 empleados.

\begin{tabular}{|c|c|c|c|c|}
\hline \multicolumn{5}{|c|}{ V04 - Conteo de número de empleados por empresa } \\
\hline $\begin{array}{c}\text { Número de } \\
\text { empleados }\end{array}$ & $\begin{array}{c}\text { Número de empresas según } \\
\text { cantidad de empleados }\end{array}$ & Porcentaje & $\begin{array}{c}\text { Conteo acumulado de } \\
\text { empresas }\end{array}$ & $\begin{array}{c}\text { Porcentaje acumulado de } \\
\text { empresas }\end{array}$ \\
\hline 1 & 161 & 15,71 & 161 & 15,71 \\
\hline 2 & 418 & 40,78 & 579 & 56,49 \\
\hline 3 & 184 & 17,95 & 763 & 74,44 \\
\hline 4 & 88 & 8,59 & 851 & 83,02 \\
\hline 5 & 51 & 4,98 & 902 & 88 \\
\hline 6 & 31 & 3,02 & 933 & 91,02 \\
\hline 7 & 19 & 1,85 & 952 & 94,05 \\
\hline 8 & 12 & 1,17 & 964 & 94,63 \\
\hline 9 & 6 & 0,59 & 970 & 95,8 \\
\hline 10 & 12 & 1,17 & 982 & 96,1 \\
\hline 11 & 3 & 0,29 & 985 & 96,49 \\
\hline 12 & 4 & 0,39 & 989 & \\
\hline
\end{tabular}




\begin{tabular}{|c|c|c|c|c|}
\hline 13 & 5 & 0,49 & 994 & 96,98 \\
\hline 14 & 2 & 0,2 & 996 & 97,17 \\
\hline 15 & 7 & 0,68 & 1003 & 97,85 \\
\hline 18 & 2 & 0,2 & 1005 & 98,05 \\
\hline 19 & 1 & 0,1 & 1006 & 98,15 \\
\hline 20 & 4 & 0,39 & 1010 & 98,54 \\
\hline 22 & 3 & 0,29 & 1013 & 98,83 \\
\hline 23 & 1 & 0,1 & 1014 & 98,93 \\
\hline 24 & 1 & 0,1 & 1015 & 99,02 \\
\hline 26 & 1 & 0,1 & 1016 & 99,12 \\
\hline 28 & 1 & 0,1 & 1017 & 99,22 \\
\hline 30 & 2 & 0,2 & 1019 & 99,41 \\
\hline 31 & 1 & 0,1 & 1020 & 99,51 \\
\hline 38 & 1 & 0,1 & 1021 & 99,61 \\
\hline 45 & 1 & 0,1 & 1022 & 99,71 \\
\hline 50 & 3 & 0,29 & 1025 & 100 \\
\hline $\mathrm{N}=$ & 1025 & & & \\
\hline
\end{tabular}

Fuente. Elaboración propia.

Por otra parte, aplicando el análisis descriptivo a las variables relacionadas en el área de la «Percepción de la Gerencia», se evidencian frecuencias de respuestas similares en las variables V05, V06, V07, V09; sin embargo, esta tendencia no se observa en la variable V10, ya que se alcanzan valores más equilibrados en las respuestas dadas, como se evidencia en la gráfica a continuación.

Figura 4. Percepción de la gerencia

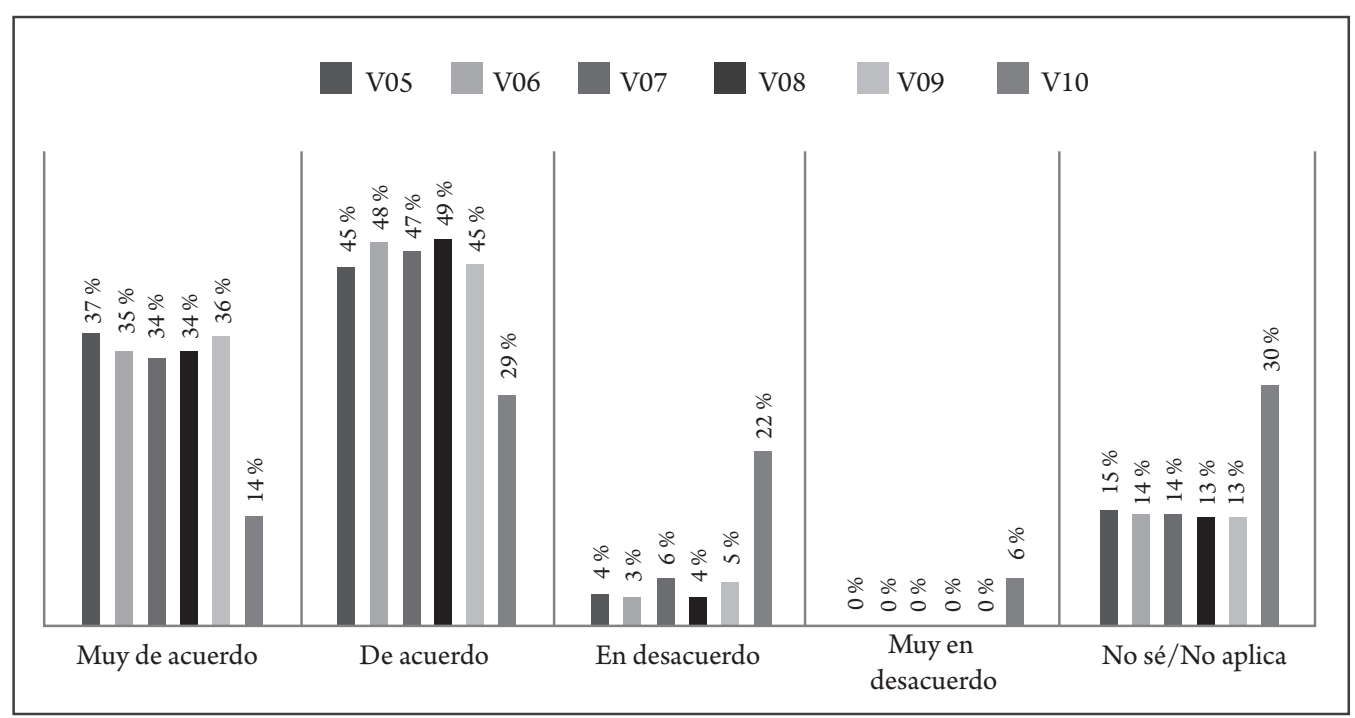

Fuente. Elaboración propia. 
Con referencia a la percepción de honestidad (V05) por parte de los colaboradores desde el punto de vista de los directivos, se pudo establecer que el $82 \%$ considera que los colaboradores son honestos, mientras que el $18 \%$ considera que no lo son.

Frente a la percepción de la responsabilidad (V06) de los colaboradores con respecto a las funciones que realizan, por parte de los directivos de la organización se observa una percepción favorable al establecer que los colaboradores son responsables en un $83 \%$ en contraposición a un $17 \%$ que considera que no son responsables.

En relación a la percepción de lealtad (V07) por parte de los colaboradores a la empresa, los directivos en un porcentaje del $81 \%$ consideran que los colaboradores son leales, y un $19 \%$ considera que tiene comportamientos desleales.

En relación a la percepción de capacidad (V08) por parte de los colaboradores a la empresa, los directivos en un porcentaje del $83 \%$ consideran que los colabo- radores cuentan con las habilidades y destrezas para realizar sus labores y un porcentaje menor del $17 \%$ considera que no tienen la capacidad para cumplir sus funciones.

En relación a la percepción del clima laboral (V08) que se maneja en la empresa, los directivos en un porcentaje del $81 \%$ consideran que se presenta buen clima laboral y en un porcentaje bajo del $19 \%$ consideran que no se cuenta con un buen clima laboral.

Frente a la percepción de salarios pagados por la empresa a los colaboradores en relación a los que se pagan en otras empresas (V10), el $42 \%$ considera que son acordes al mercado, el $30 \%$ no sabe y el $28 \%$ considera que los salarios están en desventaja en relación otras empresas.

Figura 5. V11. Enfoque de la gerencia en términos de mejorar la productividad de los colaboradores

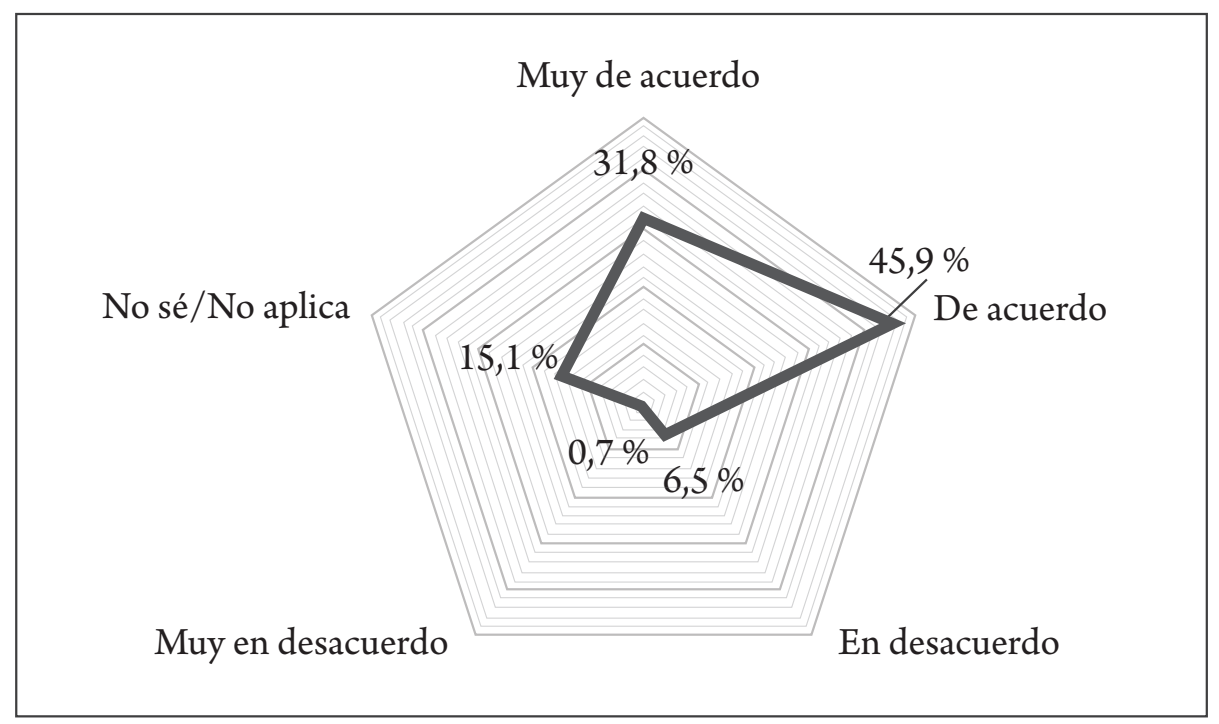

Fuente. Elaboración propia.

Finalmente, se pasa a presentar los resultados obtenidos desde el campo de las acciones implementadas por la gerencia; en ellas se observan las variables V11, V12, V13, V14 y V15. En primera medida se observa que el enfoque de la gerencia para el $78 \%$ de los encuestados presenta una perspectiva favorable, mientras el $15 \%$ sostiene que no aplica o no sabe sobre dicha condición. 
Figura 6. V12. Evaluación de satisfacción laboral

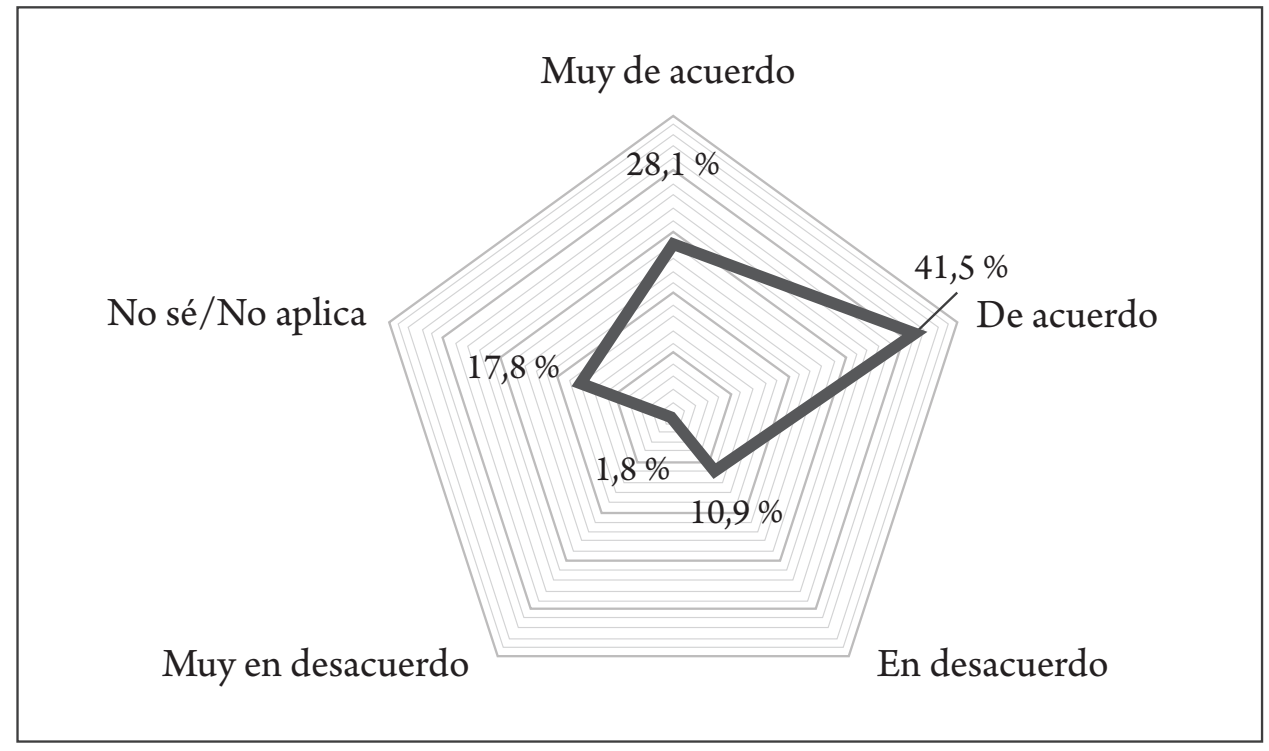

Fuente. Elaboración propia.

En seguimiento del análisis se observa, en la evaluación de satisfacción laboral que el resultado presenta un $69 \%$ de favorabilidad, seguido de un 18 \% que menciona el desconocer su aplicación y finalmente un $13 \%$ que se muestran en desacuerdo con la aplicación de esta herramienta de gestión.

Figura 7.V13. Implementación de programas para mejorar el bienestar de los colaboradores

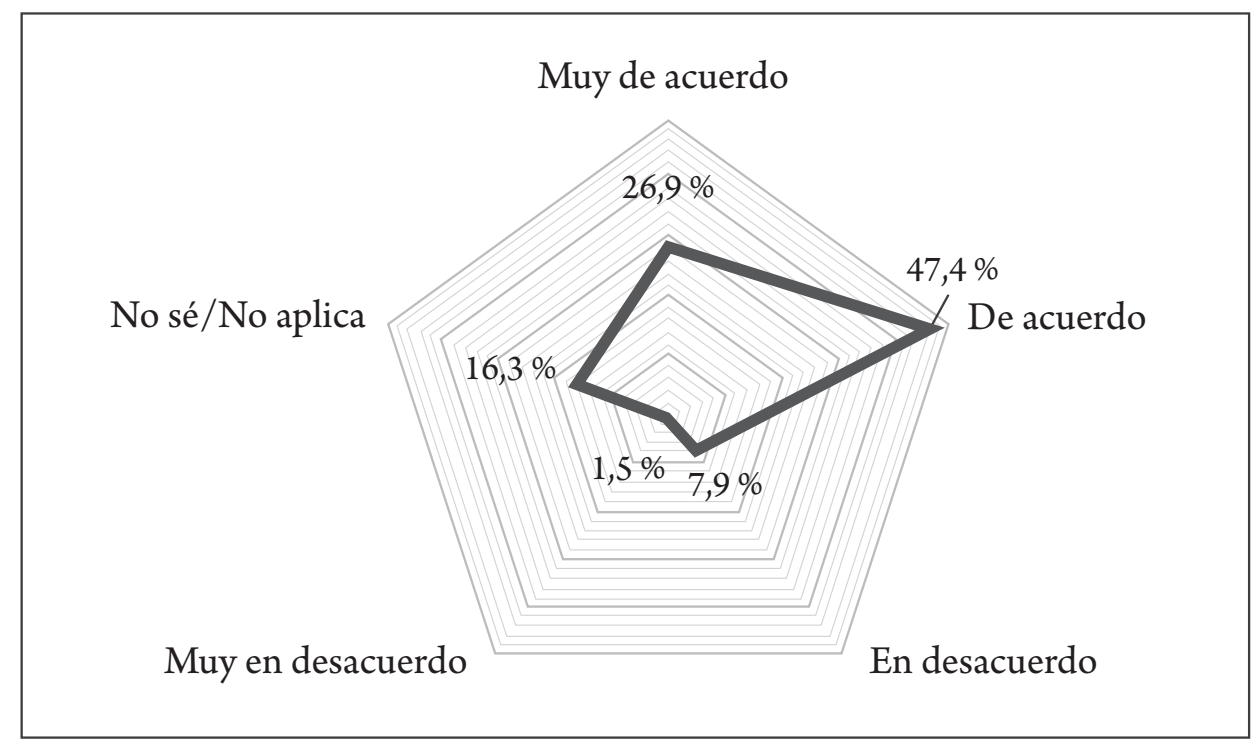

Fuente. Elaboración propia. 
Por su parte, la implementación de programas para mejorar el bienestar de los colaboradores, presenta una condición favorable en un $74 \%$, seguido de un $16 \%$ que mencionan no aplicar tal condición y un $10 \%$ que se muestra en contra de su implementación.

Figura 8. V14. Evaluación del desempeño del colaborador

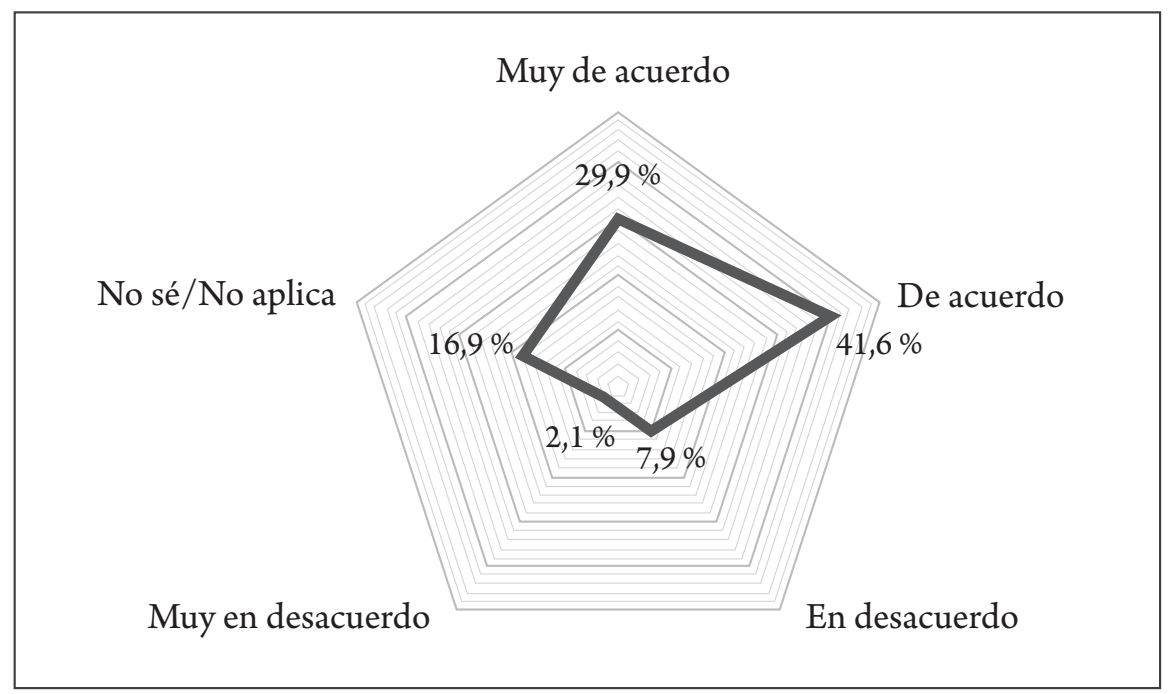

Fuente. Elaboración propia.

De igual manera, con relación a la evaluación del desempeño del colaborador, como procedimiento fundamental para la gestión del personal, se destaca que $72 \%$ de las organizaciones realizan aplicación de estas herramientas de verificación, el $17 \%$ menciona no aplicarla, mientras un $11 \%$ menciona estar en desacuerdo con su aplicación.

Figura 9. V15. Interacción de los colaboradores en el proceso de toma de decisiones de la organización

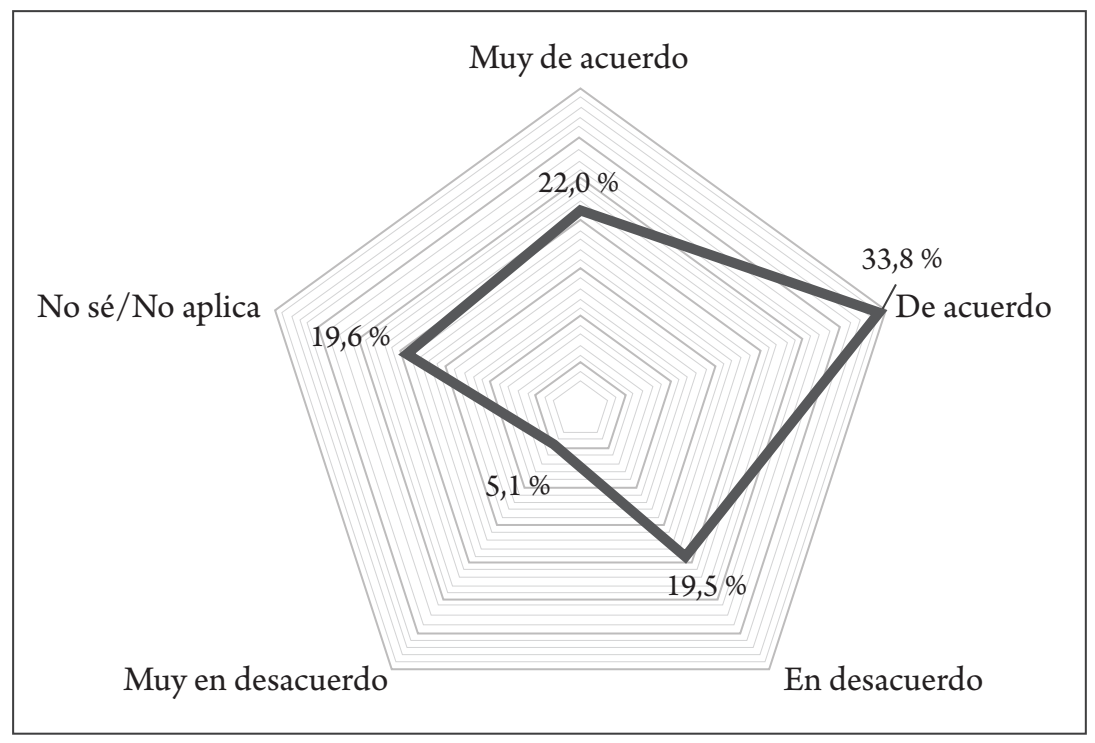

Fuente. Elaboración propia. 
Finalmente, la interacción de los colaboradores en el proceso de toma de decisiones en la organización, pretende establecer el permiso que otorga la gerencia a su personal para que participe en los procesos de toma de decisiones, de allí el $20 \%$ menciona que no aplica tal condición, el $56 \%$ acepta y favorece dicha labor, mientras el $24 \%$ mantiene al margen tal condición, así como se muestra en contra de permitir este tipo de acciones.
Una vez finalizado el análisis descriptivo, se realiza la generación de hipótesis con el fin de realizar validación estadística; dicha verificación es realizada aplicando el estadístico chi-cuadrado; las hipótesis nulas planteadas son las siguientes:

Tabla 5. Listado de hipótesis generadas para asociación de variables

\begin{tabular}{|c|l|c|}
\hline \multicolumn{2}{|c|}{ Listado de hipótesis generadas } & \multicolumn{1}{|c|}{ Descripción } \\
\hline Código hipótesis & \multicolumn{1}{|c|}{ Variables relacionadas } \\
\hline HIP1 & $\begin{array}{l}\text { La percepción de la responsabilidad de los empleados, se relaciona con el clima } \\
\text { laboral de la organización. }\end{array}$ & V6-V9 \\
\hline HIP2 & La percepción de la lealtad de los empleados y el clima laboral de la organización. & V7 - V9 \\
\hline HIP3 & $\begin{array}{l}\text { La percepción de la capacidad de los empleados y el clima laboral de la } \\
\text { organización. }\end{array}$ & V8-V9 \\
\hline HIP4 & La percepción de los salarios y el clima laboral de la organización. & V9-V10 \\
\hline HIP5 & $\begin{array}{l}\text { La percepción del clima laboral, la interacción de los empleados y las decisiones } \\
\text { que se toman al interior de la organización. }\end{array}$ & V15 \\
\hline
\end{tabular}

Fuente. Elaboración propia.

Tabla 6. Validación de las hipótesis

\begin{tabular}{|c|c|c|c|}
\hline \multicolumn{4}{|c|}{ Resultados de prueba Chi - Cuadrada de hipótesis planteadas } \\
\hline Código hipótesis & $\begin{array}{c}\text { Variables } \\
\text { relacionadas }\end{array}$ & Valor P - Pearson & Observaciones \\
\hline HIP1 & $\mathrm{V} 6-\mathrm{V} 9$ & 0,000 & $\begin{array}{l}\text { De acuerdo con los datos encontrados se concluye que las variables se } \\
\text { relacionan; por tanto, se considera estadísticamente válida la afirmación: } \\
\text { «La percepción de la responsabilidad de los empleados, se ve relacionada } \\
\text { con la percepción del clima laboral de la organización» }\end{array}$ \\
\hline HIP2 & V7 - V9 & 0,000 & $\begin{array}{l}\text { De acuerdo con los datos encontrados se concluye que las variables se } \\
\text { relacionan; por tanto, se considera estadísticamente válida la afirmación: } \\
\text { «La percepción de la lealtad de los empleados, se ve relacionada con la } \\
\text { percepción del clima laboral de la organización» }\end{array}$ \\
\hline HIP3 & $\mathrm{V} 8-\mathrm{V} 9$ & 0,000 & $\begin{array}{l}\text { De acuerdo con los datos encontrados se concluye que las variables se } \\
\text { relacionan; por tanto, se considera estadísticamente válida la afirmación: } \\
\text { «La percepción de la capacidad de los empleados, se ve relacionada con } \\
\text { la percepción del clima laboral de la organización» }\end{array}$ \\
\hline HIP4 & $\mathrm{V} 9-\mathrm{V} 10$ & 0,000 & $\begin{array}{l}\text { De acuerdo con los datos encontrados se concluye que las variables se } \\
\text { relacionan; por tanto, se considera estadísticamente válida la afirmación: } \\
\text { «La percepción de los salarios, se ve relacionada con el clima laboral de } \\
\text { la organización» }\end{array}$ \\
\hline HIP5 & V9-V15 & 0,000 & $\begin{array}{l}\text { De acuerdo con los datos encontrados se concluye que las variables se } \\
\text { relacionan; por tanto, se considera estadísticamente válida la afirmación: } \\
\text { «La percepción del clima laboral, se ve relacionada con la participación } \\
\text { en las decisiones que se toman al interior de la organización» }\end{array}$ \\
\hline
\end{tabular}

Fuente. Elaboración propia. 
A partir de la prueba Chi - Cuadrada como herramienta de validación estadística para asociación de las variables, se validaron o rechazaron las hipótesis nulas a partir de un nivel de significancia de 0,05 como valor p. Cuando este resultado sea inferior o igual se considera que existe asociación entre variables, mientras que cuando el valor sea superior se concluye que no existe relación entre variables. El análisis se realiza con apoyo del software MiniTab18, el cual es un programa diseñado para ejecutar actividades estadísticas básicas y avanzadas.

\section{Discusiones y conclusiones}

$\mathrm{U}$ na vez terminada la investigación se procede a establecer las siguientes conclusiones. Como primera medida se debe destacar la estabilidad de las respuestas de las categorías No sabe / No aplica, respecto al $15,1 \%$ que componen aquellas organizaciones con un sólo empleado, esto permite visualizar la coherencia frente a las respuestas dadas a lo largo del instrumento.

De otra parte, es importante resaltar la percepción que tienen los gerentes de sus colaboradores en aspectos que apoyan la confianza en el equipo de trabajo, esto se observa desde la comprensión de la honestidad, la responsabilidad, la lealtad, el reconocimiento de un talento humano capaz y el clima laboral.

Según Baguer (2009), el valor de la honestidad promueve el normal funcionamiento de las organizaciones, y su omisión genera el negar u ocultar responsabilidades. En el escenario de trabajo, se reconoce su fortaleza, ya que el $82 \%$ de los encuestados consideran a sus colaboradores honestos.

Por otra parte, se encuentra la responsabilidad, la cual Santos (2010) define como valor fundamental, ya que promueve el crecimiento y competitividad de la organización, condición abordada plenamente desde la perspectiva directiva, teniendo un $83 \%$ de percepción de un talento humano responsable.

Adicionalmente, se realiza un reconocimiento a la lealtad, con un $81 \%$ de favorabilidad en la percepción de colaboradores leales, esto nuevamente permite reconocer que existen bases sólidas para el desarrollo de equipos de trabajo, situación altamente favorable, entendiendo su relación con la productividad y competitividad (Prieto-Bejarano, 2013).

Finalmente, la capacidad es entendida, a nivel de talento humano, como las competencias que se tienen para resolver y tomar decisiones en una ocupación, debiendo asumir las habilidades, destrezas, experiencias y aptitudes propias de acuerdo al perfil del cargo (Redondo-Ciruelos, 2014). En este contexto, se evidencia un $83 \%$ de favorabilidad en el reconocimiento de tales condiciones.

Ahora bien, los elementos mencionados anteriormente, favorecen y explican las condiciones del clima laboral, el cual presenta un $81 \%$ de favorabilidad pues, como lo menciona Castañeda-Velasco (2016), en las mipymes tener un buen clima laboral es clave, ya que se permite al colaborador realizar sus funciones de forma adecuada, trabajando en equipo y persiguiendo los objetivos y metas organizacionales.

De acuerdo con lo anterior, el desarrollo de las mipymes en Bucaramanga y su área metropolitana, en su mayoría, presenta elementos fundamentales que reflejan la confianza existente, desde la gerencia hacia los colaboradores, contexto altamente favorable para la mejora productiva y competitiva, ya que permite el planteamiento de equipos de trabajo que podrían desarrollarse al interior de cada una de las organizaciones. 
Por otra parte, se hace necesario conocer las acciones que implementa la gerencia, esto como factor fundamental para una correcta gestión del talento humano, $y$, por ende, como un elemento que permite entender el aprovechamiento de la oportunidad observada.

Para empezar, se observa que, en el contexto abordado, sí existe un enfoque en la mejora de la productividad de los colaboradores, esto se soporta en un $77,7 \%$ de respuestas en favor de tal condición, lo que evidencia que existen procesos maduros en términos gerenciales, comprendiendo la relevancia del factor humano en la operación de la organización.

Adicionalmente, dicho grado de madurez, se soporta en la aplicación de evaluaciones de satisfacción laboral con un $69,6 \%$ de favorabilidad, en la aplicación de programas para la mejora de bienestar con $74,3 \%$ de favorabilidad, y en la aplicación de evaluaciones de desempeño con $71,4 \%$ de favorabilidad.

Continuando con lo anterior, no solamente es posible identificar un escenario de integración propicio en términos de la gestión del talento, sino que este, a su vez, está siendo aprovechado por la gran mayoría de organizaciones, a través de acciones que permiten su optimización y la generación de resultados.

Además, es relevante hacer hincapié en que evaluar el desempeño de un colaborador es un componente fundamental para las empresas, más para las mipymes por el grado de importancia regional que tienen, tal como lo establecen Díaz-Cabrera, HernándezFernaud, Isla-Díaz, Delgado-Rodríguez, Díaz-Vilela y Rosales-Sánchez (2014).

Finalmente, es relevante destacar, que a pesar de los elementos que se exponen, estos no tienen un efecto relevante en los procesos de toma de decisiones, con un $55,8 \%$ de favorabilidad, se entiende que la mipymes no han generado espacios suficientes $y$ adecuados para el desarrollo de equipos de trabajo, a pesar de contar con bases sólidas para aplicarlo.

Lo anterior permite observar que el estilo gerencial se centra principalmente desde lo autocrático, situación que resta competitividad y que además afecta el empoderamiento, lo que permite entender que, si bien se reconoce la importancia del factor humano en la empresa, su importancia se encuentra relegada a la ejecución, no a la planeación.

Sintetizando, se recomienda continuar con el desarrollo de este tipo de estudios, en especial en su aplicación en los contextos de cada empresa - micro, pequeñas y medianas empresas-, esto con el objetivo de poder observar el comportamiento de cada una de ellas y valorar posibles estrategias de mejora ajustadas a los escenarios de aplicación.

\section{Referencias}

Alles, M. A. (2005). Desarrollo del talento humano basado en competencias. Buenos Aires: Granica.

Angulo, A. (2006). Desempeño laboral y calidad de servicio de las universidades públicas del Municipio Maracaibo del Estado Zulia (Tesis de grado no publicada). Universidad Rafael Belloso Chacín. Maracaibo, Venezuela.

Aranda-Leyton, E. (2016). La gerencia de la felicidad: Un nuevo modelo para la gestión de las organizaciones (Tesis de grado). Universidad Militar Nueva Granada. Bogotá, Colombia. Recuperado de http://hdl.handle.net/10654/15964

Baguer, A. (2009). Dirección de personas: un timón en la tormenta. Madrid: Díaz de Santos.

Castañeda-Velasco, D. C. (2016). Estudio del clima laboral y su influencia en la productividad de los empleados de la empresa $\ll$ Concreteras Granizo» (Tesis degrado). Pontificia Universidad Católica del Ecuador. Quito, Ecuador. Recuperado de http:// repositorio.puce.edu.ec/handle/22000/10362

Celis-Salazar, O.; Silva-Giraldo, C. A. (2018). Nueva visión sobre la informalidad de las mipymes del área metropolitana de Bucaramanga, Santander - Causas y efectos. Revista RETO: Revista Especializada En Tecnologías Transversales De La Organización, 5(5), 23-31.

Chiavenato, Idalberto. (2007). Administración de recursos humanos. Madrid: McGraw-Hill.

Díaz-Cabrera, D.; Hernández-Fernaud, E.; Isla-Díaz, R.; DelgadoRodríguez, N.; Díaz-Vilela, L.; Rosales-Sánchez, C. (2014). Factores relevantes para aumentar la precisión, la viabilidad y el éxito de los sistemas de evaluación del desempeño laboral. Papeles del psicólogo, 35(2), 115-121.

Enciso, C. E.; Porras Jiménez, J. A. (2011). La gestión del talento humano ante el desafío de organizaciones competitivas. Gestión \& Sociedad, 4(2),167-183. 
Genesi, M.; Suarez, F. (2010). Gestión de calidad del talento humano en las organizaciones educativas inteligentes. Revista Orbis, (17), 116-155.

Jaramillo-Naranjo, O. L. (2005). Gestión del talento humano en el micro, pequeña y mediana empresa vinculada al programa Expopyme de la Universidad del Norte en los sectores de confecciones y alimentos. Pensamiento \& gestión, (18), 103137.

Mejía-Giraldo, A.; Bravo-Castillo, M.; Montoya-Serrano, A. (2013). El factor del talento humano en las organizaciones. Ingeniería Industrial, 34(1), 2-11.

Prieto-Bejarano, P. G. (2013). Gestión del talento humano como estrategia para retención del personal (Tesis de grado). Universidad de Medellín. Medellín, Colombia. Recuperado de http://hdl.handle.net/11407/160

Ramírez-Lira, E.; Dávila-Ibarra, E. (2018). Validación de la escala para la caracterización de la cultura organizacional en mipymes. Psicología Desde El Caribe, (35), 22-44. DOI: https://doi.org/10.14482/psdc.35.4.658.001
Rangel-Pico, A.; Silva-Giraldo, C.; Nino-Liévano, F.; PérezOlmos, L. (2017). Análisis del estrés en la alta gerencia y su dinámica en el proceso productivo de las mipymes en Bucaramanga, Santander (Colombia). Ide@s CONCYTEG, 12(165), 5-15.

Redondo-Ciruelos, V. (2014). Discapacidad y capacidad laboral. Medicina y Seguridad del Trabajo, 60(1), 189-195.

Santos, A. C. (2010). Gestión de talento humano y del conocimiento. Bogotá: Ecoe Ediciones.

Silva-Giraldo, C. A.; Dugarte-Mendoza, J. S.; Rueda-Mahecha, Y. M. (2020). La gerencia de proyectos desde el área de gestión humana para la disminución del riesgo biomecánico en las empresas del sector avícola en Santander, Colombia. Revista de Investigación en Gestión Industrial, Ambiental, Seguridad Y Salud en el Trabajo - GISST, 1(1), 43-67. 